\title{
Peri-Operative Communication with Patients who have Mental Health Illness or Autism: The Need for Communication Skills Training for Anaesthetists
}

\author{
Noamaan Wilson-Baig* \\ Department of Anaesthesia, Preston Royal Infirmary, UK
}

\author{
Keywords \\ Peri-operative, Communication, Mental health, Autism, Communication skills training
}

\section{Introduction}

The peri-operative period can be stressful, and patients can feel vulnerable [1]. This vulnerability can be exacerbated in patients with Mental Health Illness (MHI) or autism. Therefore, as an anaesthetist it is essential to exercise good communication with patients during the peri-operative period [1]. Compared with other specialties, the anaesthetist has a variable relationship with the patient peri-operatively. This can be divided into three phases, firstly the pre-operative assessment, secondly the peri-procedural period, and finally the post-operative period [2]. In order for the second and third phases to occur successfully, the first phase is essential as it is the first encounter the patient has with the anaesthetist. This is a time for the anaesthetist to develop a rapport with the patient. Klafta and Roizen [2] identified six interrelated goals in the pre-operative assessment. These are, firstly, to assess the health of the individual and whether anaesthesia will be appropriate. Second is to agree on a shared anaesthetic plan and discuss the anaesthetic process. The third is to mitigate the effects of anxiety, both psychologically and physiologically. The fourth is to plan how post-operative care will be conducted and what measures will be taken to ensure pain management is optimal. The fifth is to coordinate the anaesthetic process of the patient to ensure positive outcomes. The sixth is to gain informed consent for anaesthesia. Each goal requires a skilled communicator to reassure the patient that they will be cared for adequately for a procedure that the patient will be undergoing. This is especially important if the third interrelated goal is to be achieved [2]. Additionally, there are organisational pressures to achieve these goals in a time-restricted manner as theatre time is at a premium. It is therefore imperative for the anaesthetist to develop skills in effectively communicating with patients from all backgrounds.

Communication skills are generally taught at medical school. Over the years this has formed the bedrock of the curriculum. The emphasis on good communication from within the medical profession stemmed from the guidance provided by the GMC in its document, Tomorrows Doctors [3]. Doctors are taught how to use good communication skills to facilitate the patient's narrative and elucidate the patient's current understanding, concerns and expectations [4]. The information gathered is then interpreted by the doctor and a shared management plan agreed upon. The aim is that medical students would be exposed to patients from a variety of backgrounds where they could hone their communication skills. These skills would also be regularly assessed through rigorous examination.

The benefits to physician communication and patient adherence have been well documented. A meta-analysis [5] concluded that communication highly correlates with improved patient adherence, and that training doctors to communicate enhances patient adherence. The odds of patient adherence are 1.62 times higher in a doctor who has received training in communication than one who has not [3]. A criticism of this study is that it excluded studies of psychiatric care. It is also not clear if patients with autism were considered.

Patients with $\mathrm{MHI}$ or autism do not always concord to

*Corresponding author: Noamaan Wilson-Baig, Department of Anaesthesia, Preston Royal Infirmary, ST6 Academic Trainee in Anaesthesia, North West Deanery, UK

Accepted: January 25, 2020

Published online: January 27, 2020

Citation: Wilson-Baig N (2020) Peri-Operative Communication with Patients who have Mental Health Illness or Autism: The Need for Communication Skills Training for Anaesthetists. J Clin Anesth Pain Manag 4(1):92-95 
Citation: Wilson-Baig N (2020) Peri-Operative Communication with Patients who have Mental Health Illness or Autism: The Need for Communication Skills Training for Anaesthetists. J Clin Anesth Pain Manag 4(1):92-95

treatment plans. There is evidence that these patients may experience negative outcomes as a result of ineffective communication [6]. A good healthcare professional-patient partnership is the key to encouraging a patient's confidence and trust with regards to their healthcare management [6]. It is therefore essential that good communication between the healthcare professional and these patients must be fostered. For patients with $\mathrm{MHI}$ or autism, their families, or carers, this can be challenging. This can be demonstrated by the two following recent cases I was recently involved with.

\section{Case 1}

This patient was scheduled to have an urgent neurological procedure. I went to see him to discuss the anaesthesia for the procedure. I was informed that the patient suffered from severe mental health issues, which included depression and anxiety. In addition, he drank excessively and used amphetamines. I was also informed that this patient was very abusive to ward staff and threatening to take his own discharge. The surgical team was considering sectioning the patient due to his medical condition. In addition, the patient had around ten security guards in the vicinity and surrounding the patent. On arriving at the ward, I could see the patient screaming and shouting expletives at the security guards and ward staff.

As a result of my observations, I decided to take an alternative approach. I asked the security guards to leave bar two, who waited outside the bay of the ward. I then introduced myself to the patient. I noted the patient was a football fan and considering the recent success in Europe, I decided to focus the discussion on this. After a while, I felt I developed a rapport with him. I then proceeded to explore the patient's ideas and concerns with regards to his current admission. It transpired that he did not understand what was going to happen surgically and why he was nil by mouth. He told me that people were "telling me stuff I don't understand". On learning this, I took time to explain this to him whilst ensuring that I was conversing with him in terms that helped him understand the treatment he was about to receive. As a result, the patient was responsive to my conversation with him and was happy to proceed with the procedure. The security guards were then withdrawn, and no issues occurred with the staff. He came for surgery and it was uneventful as was the post-op period.

\section{Case 2}

This was an 8-yr-old child, who required an urgent general surgical procedure. I was asked to conduct an anaesthetic review. I was informed that the child is autistic and is very irritable and refusing to let the medical staff treat him. On the ward, I could hear the surgeon struggling to assess the child as the child was screaming and refusing to let the surgeon near him. When the surgeon left the patient, he wished me good luck. From the outside of the room, I noted that the child was wearing a T-shirt with a print of the Incredible Hulk. I thought I would use my knowledge of the comics to initially talk to him. I asked him if he liked the Hulk and discussed some interesting facts about the Hulk, e.g. the Hulk can jump America in three leaps. We also discussed other characters like Iron-Man,
Captain America, Spiderman and Wonder Woman. I felt that this built a good rapport between the boy and myself. As the child was with his mother, we were able to discuss the child's anaesthesia without any issues. As a result, the child was successfully anaesthetised and had an uneventful surgery.

These cases demonstrate the challenges faced by healthcare professionals when managing patients with $\mathrm{MHI}$ or autism. In these cases, a tricky situation was diffused. In both cases, I tried to apply the Calgary-Cambridge approach [4]. This was the approach I was taught at medical school. By identifying common ground with the patients, i.e. football and superheroes, I was able to build a rapport with the patient. I was then able to explore their ideas, concerns, and expectations with regards to the procedure that the patients were scheduled to have. I tried to show curiosity for them as human beings and conversed with them in terms that would help the patients understand the treatment they were going to receive. I was keen to involve them in planning the next steps with regards to the anaesthesia and arrived at a shared decision plan for them. As an anaesthetist, assessing and understanding the patient's knowledge and concerns allows me to direct the consultation in a way that would address these areas whilst providing information about the bespoke anaesthetic process. It is hoped that an agreeable shared decision would mitigate underlying anxieties the patient may have.

These cases also demonstrate the challenges faced by healthcare professionals when communicating with different age groups. The styles and strategies are distinct between these populations and require specific skill sets and training. Additionally, psychiatry is a large specialty with a variety of diagnoses which can present very differently to the case presented here. Similarly, autism is a spectrum condition that affects individuals in a variety of ways. Some autistic people may have learning disabilities, or mental health issues and will require support bespoke to them. Although it may be too simplistic to consider mental health issues and autism collectively, it is important to acknowledge that effectively communicating with patients presenting with these conditions is integral to successfully managing them during the peri-operative period.

In healthcare, patients with $\mathrm{MHI}$ or autism regularly seek assistance from services provided by the NHS. This could be specialist treatment by mental health/autism teams in the primary and secondary sector, or by specialists from other specialties in medicine. The interactions between these specialists and patients with $\mathrm{MHI}$ or autism can be challenging as the examples given demonstrate. In the case of the patient requiring a neurological intervention the presence of security guards and the inability to establish rapport with the patient may have led the patient to feel alienated. He appeared disengaged and could have deteriorated if he took his own discharge. There is evidence that this exists, and patients could be a risk to themselves and others [6]. It is therefore essential that effective communication must be exercised in order to manage these patients. This has shown to be associated with increased patient satisfaction and engagement with healthcare [7]. 
Citation: Wilson-Baig N (2020) Peri-Operative Communication with Patients who have Mental Health Illness or Autism: The Need for Communication Skills Training for Anaesthetists. J Clin Anesth Pain Manag 4(1):92-95

The Tomorrows Doctors [3] document specifically states that,

Students must have opportunities to practise communicating in different ways, including spoken, written and electronic methods. There should be guidance about how to cope in difficult circumstances.

An example provided is communicating with and treating patients with severe mental or physical disabilities.

Although undergraduate medical students undergo excellent communication skills training (CST) through role-playing and lectures, the training for specific patient groups may not be sufficient enough to prepare the practitioner for managing these patients, e.g. patients with $\mathrm{MHI}$ or autism. Consideration must, therefore, be given as to whether CST for managing patients with $\mathrm{MHI}$ or patients with autism should be provided to medical students, and not be just restricted to the psychiatric module. Consideration must also be given to continuing CST during foundation training and the core training years of the doctor's chosen specialty. Currently, this only happens with doctors undergoing training for general practice. For example, the BATHE model [8] is encouraged for psychotherapeutic consultations. This model is useful for general practitioners as it aims to screen for mental health issues, develop a connection between the patient and clinician, and support to empower patients to manage the situation they are currently in. General practitioners are taught how to address the patient's Background, Affect, Troubles, Handling of their situation, followed by an Empathetic response (BATHE). The POEMS course (Positive Outcome and Experience Management Strategies) is a one-day course set up by a UK base charity [9]. The aim of this course is to train professionals to manage anxious children undergoing medical procedures. However, the main focus is on identifying and managing peri-operative anxiety in children. Autism affects both adults and children and may require specialist and distinctive training.

A Cochrane review [10] to assess the effects of interventions to promote patient-centred care approaches to clinical consultations showed most studies to be directed at primary care. Additionally, these interventions were found to be effective and that short-term training (less than 10 hours) is as successful as longer training. However, the review showed mixed results when patient satisfaction, health behaviour, and health status were considered. Papageorgiou, et al. [11] conducted a Cochrane review to examine the effectiveness of CST for mental health professionals who work with people with severe mental health illness. The studies examined in this review were of low quality and therefore conclusions could not be drawn as to the efficacy of CST in mental healthcare professionals. With regards to autism, studies have demonstrated inadequate training for healthcare providers with little focus on adults with autism [12,13]. There are no studies involving anaesthetists and patients with $\mathrm{MHI}$ or autism.

Due to the lack of evidence-based studies, research must be conducted to firstly perform a qualitative study in patients with $\mathrm{MHI}$ or autism to assess their experiences and attitudes to undergoing surgery and anaesthesia. Although there is some evidence with regards to what patients understand towards the anaesthetic process [1,2], what is not clear is how the mode of communication to patients with $\mathrm{MHI}$ or autism can be optimally provided. This reflection does acknowledge that it may be too simplistic to consider mental health issues and autism collectively. However, there has to be a starting point which can be achieved through research.

Currently, anaesthetists, like specialists in other specialties develop their own styles of communication with experience [1]. This includes both verbal and non-verbal communication. Despite undergoing training in psychiatry and developmental disorders as an undergraduate, the ability to effectively manage these patients could be dependent on the exposure to this population group. Anaesthetists' do not manage patients with $\mathrm{MHI}$ or autism to the same level as a specialist working in these areas. Considering the inconclusive data from the review by Papageorgiou, et al. [11], one can only imagine what the situation is likely to be in non-mental health specialties.

\section{Acknowledgments}

I would like to thank Professor Alexia Papageorgiou, Professor of Clinical Communication and Chair of the Centre of Medical Education at the University of Nicosia Medical School for kindly reviewing the manuscript.

\section{Conflicts of Interests}

None declared.

\section{Funding}

No funding was received.

\section{References}

1. Smith AF, Mishra K (2010) Interaction between anaesthetists, their patients, and the anaesthesia team. Br J Anaesth 105: 6068.

2. Klafta JM, Roizen MF (1996) Current understanding of patients' attitudes toward and preparation for anesthesia: A review. Anesth Analg 83: 1314-1321.

3. Rubin P, Franchi-Christopher D (2002) New edition of Tomorrow's Doctors. Medical Teacher 24: 368-369.

4. Kurtz SM, Silverman JD (1996) The calgary-cambridge referenced observation guides: An aid to defining the curriculum and organizing the teaching in communication training programmes. Med Educ 30: 83-89.

5. Zolnierek KB, Dimatteo MR (2009) Physician communication and patient adherence to treatment: A meta-analysis. Med Care 47: 826-834.

6. Priebe S, Watts J, Chase M, et al. (2005) Processes of disengagement and engagement in assertive outreach patients: Qualitative study. Br J Psychiatry 187: 438-443.

7. McCabe R, Heath C, Burns T, et al. (2002) Engagement of patients with psychosis in the medical consultation: A conversation analytic study. BMJ 325: 1148-1151.

8. Lieberman JA $3^{\text {rd }}$, Stuart MR (1999) The BATHE Method: Incorporating counseling and psychotherapy into the everyday man- 
Citation: Wilson-Baig N (2020) Peri-Operative Communication with Patients who have Mental Health Illness or Autism: The Need for Communication Skills Training for Anaesthetists. J Clin Anesth Pain Manag 4(1):92-95

agement of patients. Prim Care Companion J Clin Psychiatry 1: 35-38.

9. (2019) POEMS For Children.

10. Dwamena F, Holmes-Rovner M, Gaulden CM, et al. (2012) Interventions for providers to promote a patient-centred approach in clinical consultations. Cochrane Database Syst Rev.

11. Papageorgiou A, Loke YK, Fromage M (2017) Communication skills training for mental health professionals working with people with severe mental illness. Cochrane Database Syst Rev.

12. Shattuck PT, Roux AM, Hudson LE, et al. (2012) Services for adults with an autism spectrum disorder. Can J Psychiatry 57: 284-291.

13. Nicolaidis C, Raymaker DM, Ashkenazy E, et al. (2015) "Respect the way I need to communicate with you": Healthcare experiences of adults on the autism spectrum. Autism 19: 824-831. 